\title{
Disruption of Nectin-Like 1 Cell Adhesion Molecule Leads to Delayed Axonal Myelination in the CNS
}

\author{
Jinsil Park, ${ }^{2 *}$ Ben Liu,,${ }^{1,2 *}$ Tao Chen, ${ }^{1}$ Hong Li, ${ }^{2}$ Xuemei Hu, ${ }^{2}$ Jing Gao, ${ }^{1}$ Ying Zhu, ${ }^{2}$ Qiang Zhu, ${ }^{2}$ Boqin Qiang, ${ }^{1}$ \\ Jiangang Yuan, ${ }^{1}$ Xiaozhong Peng, ${ }^{1}$ and Mengsheng Qiu ${ }^{2,3}$ \\ ${ }^{1}$ The National Laboratory of Medical Molecular Biology, Institute of Basic Medical Sciences, Chinese Academy of Medical Sciences and Peking Union \\ Medical College, Beijing 100005, China, ${ }^{2}$ Department of Anatomical Sciences and Neurobiology, School of Medicine, University of Louisville, Louisville, \\ Kentucky 40292, and ${ }^{3}$ Institute of Urological Surgery, Tianjin Medical University, Tianjin 300211, China
}

Nectin-like 1 (Necl-1) is a neural-specific cell adhesion molecule that is expressed in both the CNS and PNS. Previous in vitro studies suggested that Necl-1 expression is essential for the axon-glial interaction and myelin sheath formation in the PNS. To investigate the in vivo role of Necl-1 in axonal myelination of the developing nervous system, we generated the Necl-1 mutant mice by replacing axons 2-5 with the LacZ reporter gene. Expression studies revealed that Necl-1 is exclusively expressed by neurons in the CNS. Disruption of Necl-1 resulted in developmental delay of axonal myelination in the optic nerve and spinal cord, suggesting that Necl-1 plays an important role in the initial axon-oligodendrocyte recognition and adhesion in CNS myelination.

Key words: cell adhesion molecule; gene targeting; myelination; spinal cord; optic nerve; knock-out

\section{Introduction}

Nectin-like (Necl) molecules are members of the synaptic cell adhesion molecule family (SynCAM) consisting of four members, Necl1-4 (Takai et al., 2003; Biederer 2006). All four members contain three extracellular Ig-like domains, a single transmembrane domain, and a short cytosolic tail that interacts with protein 4.1 and PDZ (PSD-95/Dlg/ZO-1)-domain proteins. The NECL proteins mediate $\mathrm{Ca}+$ independent cell adhesion by homophilic and/or heterophilic interactions and are highly enriched in the synaptic terminals (Biederer et al., 2002; Fogel et al., 2007). Necl-1 (SynCAM3) is exclusively expressed in the neural tissues in both the PNS and CNS (Fukuhara et al., 2001; Takai et al., 2003; Kakunaga et al., 2005; Zhou et al., 2005). Necl-2 (SynCAM1 or RA175) and Necl-3(SynCAM2) are mostly transcribed in the neural tissues, but their expression can also be detected in other tissues such as lung and testis (Urase et al., 2001; Wakayama et al., 2001; Fujita et al., 2005; Pellissier et al., 2007). Necl-4 (SynCAM4) transcript is detected in both neurons and glial cells (Fogel et al., 2007; Maurel et al., 2007; Spiegel et al., 2007). Immunohistochemical staining revealed that NECL-1-4 adhesion proteins are all distributed to the axonal surfaces, and are highly enriched at various contact sites among axons (Biederer et al., 2002; Kakunaga et al., 2005; Pellissier et al., 2007). The localization of these NECL proteins suggests their possible involvement in axonal path-

\footnotetext{
Received June 10, 2008; revised Sept. 4, 2008; accepted 0ct. 18, 2008.

This work was supported by grants from the National Multiple Sclerosis Society (RG 3275) and National Institutes of Health (R01 NS37717), the National Natural Sciences Foundation of China (Grant No. 30828004, 30721063); "973” project, China (No. 2005CB522507); and Program for New Century Excellent Talents in University, China (No. NCET07-0505). We thank Dr. Martha Bickford and Kathy Campbell for assistance with electron microscopy.

*J.P. and B.L. contributed equally to this work.

Correspondence should be addressed to either of the following: Xiaozhong Peng, at the above address; E-mail: pengxiaozhong@pumc.edu.cn; or Mengsheng Qiu, at the above address; E-mail: m0qiu001@louisville.edu. DOI:10.1523/JNEUROSCI.2665-08.2008

Copyright $\odot 2008$ Society for Neuroscience ～0270-6474/08/2812815-05\$15.00/0
}

finding, fasciculation, synapse assembly during early neural development (Takai et al., 2003; Sara et al., 2005; Miyoshi and Takai, 2007).

Recently, Necl-1 and Necl-4 molecules have been implicated in the control of axon-glial interactions and myelin sheath formation in the PNS. In the PNS, NECL-1 protein is localized on the axonal surface membrane of internodes and paranodes. NECL-4 (SynCAM4) protein is predominantly found in myelinating Schwann cells, particularly on paranodal loops and the adaxonal membrane (apposing the axon) of myelin sheath (Maurel et al., 2007; Spiegel et al., 2007). The heterophilic binding between Necl-1 and Necl-4 was shown to be indispensable for in vitro myelination of PNS neurons by Schwann cells. Disruption of Necl-1 and Necl-4 interaction with soluble extracellular domains, RNAi or dominant negative forms dramatically inhibited axonal myelination in the coculture of DRG neurons and Schwann cells (Maurel et al., 2007; Spiegel et al., 2007). Based on these observations, it was proposed that axonal myelination is mediated by the heterophilic binding between the axonal NECL-1 and glial NECL-4 proteins (Perlin et al., 2007).

To investigate the in vivo role of Necl-1 in axonal myelination, we inactivated the mouse Necl-1 molecule by the standard gene targeting approach. The mutant mice were viable and fertile, but displayed a developmental delay of axonal myelination in the developing CNS (optic nerve and spinal cord). Surprisingly, myelination of sciatic nerve in the PNS did not appear to be affected by the Necl-1 mutation. The relatively mild myelination phenotype in the mutant animals has suggested a possible functional compensation from other adhesion molecules.

\section{Materials and Methods}

Gene targeting. A BAC clone containing the Necl-1 genomic DNA was purchased from Invitrogen. The gene targeting vector was constructed by replacing axons $2-5$ with $\beta$-galactosidase reporter gene and the neomy- 
cin resistance gene. Targeting vector was subsequently linearized and introduced into ES cells by electroporation. Genomic DNA isolated from ES clones was digested with BamHI and $K p n I$ before hybridization with the $3^{\prime}$ flanking probe as indicated in Figure $2 A$. The wild-type allele gave a band of $10 \mathrm{~Kb}$, whereas the targeted allele produced a band of $6.0 \mathrm{~Kb}$ (see Fig. $2 \mathrm{~B}$ ). Mutant ES clones were characterized and injected into C-57BL blastocysts to produce chimeric mice or germline transmission. Homozygous mice derived from these two independent lines showed the same phenotype.

For genotyping by PCR, three specific primers were used simultaneously in the same reaction (see Fig. 2). Primer P3 (GCTATTCGGCTATGACTGGG) and primer P5 (AGACATGGAGACAAAGGCTCT) amplified a $1.0 \mathrm{~Kb}$ fragment from Neo to the $3^{\prime}$ arm in the mutant allele, whereas primer P4 (ATCTGAAGGGTCCAGACAATATC) and primer P5 amplified a $0.5 \mathrm{~Kb}$ fragment from the wild-type allele (see Fig. 2C). The PCR conditions are $95^{\circ} \mathrm{C}$ for $5 \mathrm{~min} ; 35$ cycles of $94^{\circ} \mathrm{C}$ for $30 \mathrm{~s}, 56^{\circ} \mathrm{C}$ for $30 \mathrm{~s}, 72^{\circ} \mathrm{C}$ for $2 \mathrm{~min}$, followed by incubation at $72^{\circ} \mathrm{C}$ for $10 \mathrm{~min}$.

Animal care and experimental procedures conformed to National Institutes of Health guidelines and were approved by the Institutional Animal Care and Use Committee at the University of Louisville (protocol \# 05143), Louisville, KY.

In situ RNA hybridization and immunostaining. Animals were deeply anesthetized and perfused with $4 \%$ paraformaldehyde (PFA), and tissues were isolated and postfixed in $4 \%$ PFA at $4{ }^{\circ} \mathrm{C}$ overnight. Fixed spinal cord and brain tissues were embedded in OCT medium and sectioned on a cryostat. Frozen sections ( $16 \mu \mathrm{m}$ thick) were subject to in situ hybridization with digoxigenin-labeled riboprobes according to Schaeren-Wiemers and Gerfin-Moser (1993) with minor modifications.

For immunofluorescent staining, tissue sections were incubated with the primary antibodies at the following dilutions: anti-NeuN (1:1000, Millipore), anti-GFAP (1:1000; Millipore) or anti-CC1 (1:300; Oncogene Science) antibodies. Alexa-488- or Alexa 596-conjugated secondary antibodies were obtained from Invitrogen and used at 1:200 dilution. Anti-NeuN immounohistochemistry was performed in conjunction with the VECSTAIN ABC staining kit (Vector) with DAB substrate according to the manufacture's protocol.

Western immunoblotting. Brain, spinal cord or sciatic nerve tissues were isolated from postnatal wild-type, heterozygous and homozygous animals and lysed in SDS-PAGE sample buffer. $15 \mu \mathrm{g}$ of protein from each genotype was loaded for electrophoresis and subsequently detected with rabbit polyclonal anti-Necl-1 antibody (against the cytoplasmic domain) or anti-MBP antibody (Millipore) according to the standard protocol. Anti- $\beta$-actin antibody was used as an internal control (Sigma).

Ultrastructural analyses of myelin structures. Wild-type and Necl-1 mutant littermates from various age groups [postnatal day 7 (P7), P15, P60] were cardially perfused with $3 \%$ glutaraldehyde in $0.1 \mathrm{M}$ cacodylate buffer, $\mathrm{pH} 7.2$, and small pieces of tissues from the optic nerve, the ventral white matter of the spinal cord (at T6 level), and the sciatic nerves were removed and postfixed for three additional hours. Tissues were then washed several times with cacodylate buffer, postfix in $1 \%$ osmium tetroxide for $1 \mathrm{~h}$, washed again with the buffer before dehydration through a series of graded alcohol. Fixed tissues were subsequently embedded in epony plastic and sectioned at $800-1000 \AA$ on a diamond knife and mount on 200 mesh copper grids. Ultra thin sections were stained with uranium acetate and lead citrate, and examined under a Philips CM10 EM operating at $60 \mathrm{kV}$.

For statistical analyses of axonal myelination, the number of axons that were wrapped by compact myelin sheaths was calculated from each micrograph for each genotype $(n=3)$. Statistical significance ( $p$ value) was assessed by Student's $t$ test.

\section{Results}

\section{Targeted disruption of mouse Necl-1 gene}

To determine the role of $\mathrm{Necl}-1$ in axonal myelination, we inactivated $\mathrm{Necl}-1$ by homologous recombination in embryonic stem (ES) cells. The targeting vector was designed to replace axons 2-5 with the $\beta$-galactosidase gene and the Neo cassette (Fig. $1 A$ ). Following electroporation and drug selection, 200 independent ES clones were analyzed by genomic Southern blot with the 3' flanking probe. Eight clones harboring homologous recombination were obtained. Two mutant ES clones with correct karyotypes were injected into blastocysts from C57BL mice and both clones transmitted the mutant allele through germ line. Mutant animals were identified by either Southern blotting (Fig. $1 B$ ) or by PCR analysis (Fig. 1C). Genotypic analysis of the offspring from the heterozygous matings showed typical Mendelian segregation ratio (36:74:34), indicating that the Necl-1 mutation does not cause lethality. Homozygous mice were viable and fertile, and morphologically indistinguishable from their heterozygous and wild-type littermates.

The disruption of the Necl-1 gene in homozygous mutants was confirmed by the lack of Necl-1 in situ signal on P7 mutant spinal cord tissues (data not shown), and by the absence of protein expression in anti-NECL-1 Western immunoblotting (Fig. $1 D)$. The anti-NECL-1 antibody recognized a single protein of 45 $\mathrm{Kd}$ in the wild-type and heterozygous spinal cord, but not in the homozygous tissues, indicating that the mutant allele is null.

\section{Necl-1 is exclusively expressed in CNS neurons}

To examine the dynamic expression of $\mathrm{Necl}-1$ in details, we performed the LacZ staining and Necl-1 in situ signal on adjacent 

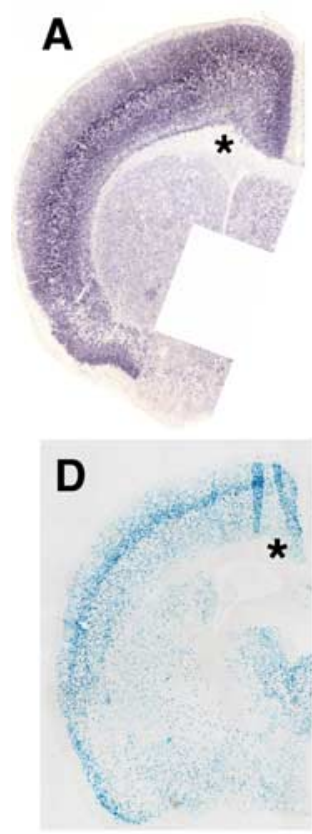

Retina

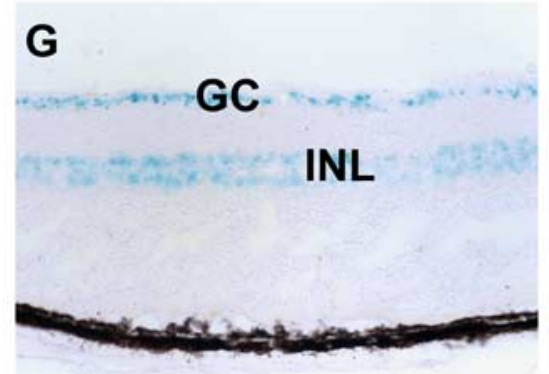

Cerebellum

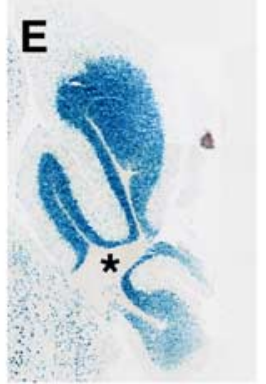

G
Cortex

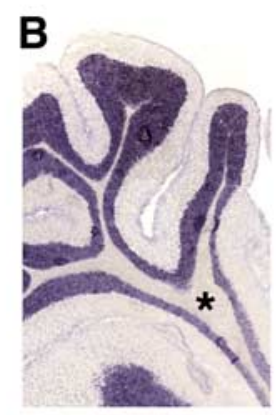

gure 2. Restrictive expression of Necl- 7 in adult CNS neurons. $\boldsymbol{A}-\boldsymbol{F}$, Cerebral, spinal and cerebellar tissues were isolated from Necl-1 1 heterozygous animals and subjected to in situ RNA hybridization with Necl-1 probe $(\boldsymbol{A}-\boldsymbol{C})$ or LacZ staining $(\boldsymbol{D}-\boldsymbol{F})$. Asterisks indicate the lack of Necl-1 expression in the white matter of the cerebral cortex $(\boldsymbol{A}, \boldsymbol{D})$, cerebellum $(\boldsymbol{B}, \boldsymbol{E})$ and spinal cord $(\boldsymbol{C}, \boldsymbol{F}) . \boldsymbol{G}$, LacZ staining in retinal ganglionic neurons (GC) and inner nuclear layer (INL) in the adult heterozygous mice. $\boldsymbol{H}$, Double labeling of LacZ signal and anti-NeuN immunohistochemical staining.

slide sections of various adult CNS tissues in heterozygous animals. Our results revealed the nearly identical patterns of LacZ histochemical staining and the endogenous $\mathrm{Necl}-1$ expression in the gray matter of the cerebral cortex, cerebellum and spinal cord (Fig. $2 A-F$ ), indicating that $\beta$-galactosidase reporter gene faithfully reflects that of the endogenous Necl-1 gene. Detailed expression studies in the developing spinal cord confirmed that Necl-1 expression was restricted to the gray matter throughout animal development (supplemental Fig S1, available at www.jneurosci. org as supplemental material). The lack of LacZ staining or Necl-1 signal in the white matter regions indicated that $\mathrm{Necl}-1$ is not transcribed in glial cells. In the retina, LacZ staining was detected in ganglionic neurons and the inner nuclear layer consisting of bipolar, horizontal and amacrine interneurons (Fig. $2 G$ ). Neuron-specific expression of $\mathrm{Necl}-1$ was further verified by the double labeling experiments showing that all LacZ + cells in the spinal cord coexpressed NeuN (Fig. $2 H$ ), a specific marker for postmitotic differentiated neurons in the CNS (Mullen et al., 1992; Sarnat et al., 1998).

\section{Necl-1 mutation caused a} developmental delay of axonal myelination in the CNS

The selective expression of $\mathrm{Necl}-1$ in the CNS neurons and axonal surface has raised the possibility that $\mathrm{Necl}-1$ may also mediate the axon-oligodendrocyte recognition and adhesion in CNS myelination. To examine this possibility, we first analyzed the myelin ensheathment in the optic nerves by electronic microscopy (EM). The optic nerve has been widely used as a myelination model for the developing CNS, as it is predominantly composed of myelinated axons and devoid of neuronal cell bodies. At P7, a small percentage of optic nerves started to be wrapped by myelin sheath in the wild-type pups (Fig. $3 A$ ). In contrast, myelin structures were completely absent in the mutant nerves (Fig. $3 B)$. At P15, myelin sheaths were found in the mutant nerves as well, but the number of myelinated axons was significantly smaller than that in the wild-type nerves (Fig. 3C,D). However, in adult tissues (P60), there was no significant difference in the number of myelinated axons and the thickness of myelin sheaths around axons (Fig. 3E,F). These results suggested that myelination in the optic nerves was initially delayed by the Necl-1 mutation, but the deficit was overcome at later stages.

The developmental delay of axonal myelination was similarly observed in the postnatal mutant spinal cords. At both P7 and P15, axon myelination in the ventrolateral white matter was significantly reduced in mutant tissues (Fig. 4A-D). However, at $\mathrm{P} 60$, myelination in the spinal cord was comparable between the wildtype and mutant littermates (Fig. 4E,F). The delayed axonal myelination did not appear to be caused by defective differentiation of neurons or glia, as suggested by the normal expression of mature markers for neurons, oligodendrocytes and astrocytes in P7 mutant spinal cords (supplemental Fig S2A-F, available at www.jneurosci.org as supplemental material). Normal differentiation of oligodendrocytes was further confirmed by Western immunoblotting, which revealed a comparable level of expression of myelin basic protein (MBP) in postnatal wild-type and mutant spinal cords (supplemental Fig S2G, available at www.jneurosci.org as supplemental material).

Previous studies demonstrated that heterophilic binding between the neuronal NECL-1 protein and glial NECL-4 protein is essential for the myelination of PNS neurons by Schwann cells in culture (Maurel et al., 2007; Spiegel et al., 2007). Surprisingly, disruption of Necl-1 had little effect on Schwann cell maturation and the myelination of sciatic nerves. From P7 to P60, there were no significant differences in MBP expression, the percentage of myelinated axons and the thickness of myelin sheaths between the wild-type and mutant nerves (supplemental Fig S3, available at www.jneurosci.org as supplemental material). 


\section{Discussion}

$\mathrm{Necl}-1$ is a nectin-like cell adhesion molecule that is specifically expressed in the neural tissues (Zhou et al., 2005). It was previously reported that $\mathrm{Necl}-1$ is expressed by both neurons and glia in the $\mathrm{CNS}$, and is enriched in the contact sites between axons and glial processes (Takai et al., 2003; Kakunaga et al., 2005). Here, we provided evidence that $\mathrm{Necl}-1$ is exclusively expressed by neurons in the CNS. First, Necl-1 expression as detected by in situ hybridization and LacZ histochemical staining in Necl-1 heterozygous mice was restricted to the gray matters throughout the entire CNS (Fig. 2). While neuron cell bodies are located in the gray matter, glial cells can be found in both gray and white matters. Second, double labeling experiments demonstrated that LacZ+ cells coexpressed the neuronal marker NeuN (Fig. 2). Similarly, in the PNS, Necl-1 was reported to be specifically expressed by DRG neurons, but not by Schwann cells (Maurel et al., 2007; Spiegel et al., 2007). One plausible explanation for the discrepancy between this and previous study (Kakunaga et al., 2005) could be due to the potential cross-reactivity of the antibody that was used in the immunostaining experiment (Kakunaga et al., 2005), given the high degree of protein sequence similarities among the $\mathrm{NeCl}$ members.

Our studies demonstrated that Necl-1 plays an important role in the initiation of axonal myelination process in the CNS. At early postnatal stages (P7-P15), the number of myelinated axons in the optic nerve and spinal cord was markedly reduced in the mutants (Figs. 3, 4). At later stages (P60), there was an insignificant difference in the number of myelinated fibers or the thickness of myelin sheaths in both tissues between the normal and mutant adult tissues. Thus, there was an apparent developmental delay of CNS myelination in the Necl-1 null mutants. It was previously shown that axonal myelination in the CNS requires the close contacts between axon shafts and oligodendrocyte processes (Colello and Pott, 1997; Edgar and Garbern, 2004). Necl-1 could be one of the important axonal membrane factors that mediate the initial axon-oligodendrocyte interaction and adhesion. Since $\mathrm{Necl}-1$ is not expressed by oligodendrocyte cells throughout development (Fig. 2; supplemental Fig S1, available at www.jneurosci.org as supplemental material), the regulation of CNS myelination by $\mathrm{Necl}-1$ is likely to be mediated by its heterophilic interactions with other adhesion molecules that are expressed by oligodendrocytes. One potential glial partner for Necl-1 could be Necl-4, since Necl-1 and Necl-4

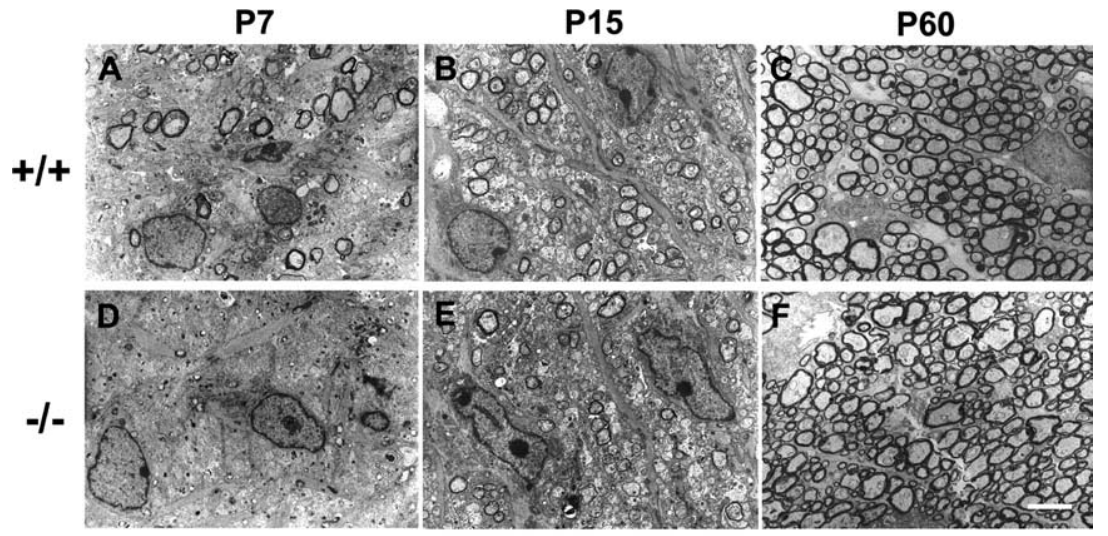

G Myelination in Necl1 KO optic nerves

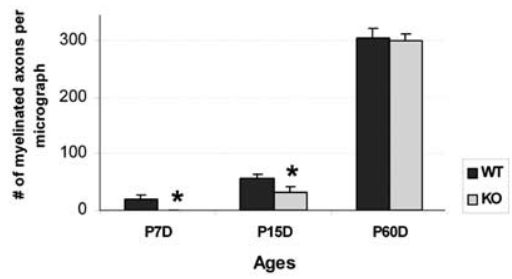

Figure 3. Delayed axonal myelination in the Necl-1 mutant optic nerves. $\boldsymbol{A}-\boldsymbol{F}$, Ultra-thin sections from P7, P15 and P60 wild-type $(\boldsymbol{A}-\boldsymbol{C})$ and Necl-1 mutant $(\boldsymbol{D}-\boldsymbol{F})$ optical nerves were examined under transmission EM. G, Number of myelinated axons per micrograph $\left(500 \mu \mathrm{m}^{2}\right)$ in the wild-type and mutant tissues at various postnatal stages $(n=3)$. Statistically significant differences ( $p$ value $<0.05$ ) between the wild-type and mutant animals were indicated by asterisks. Scale bar, $2 \mu \mathrm{m}$.

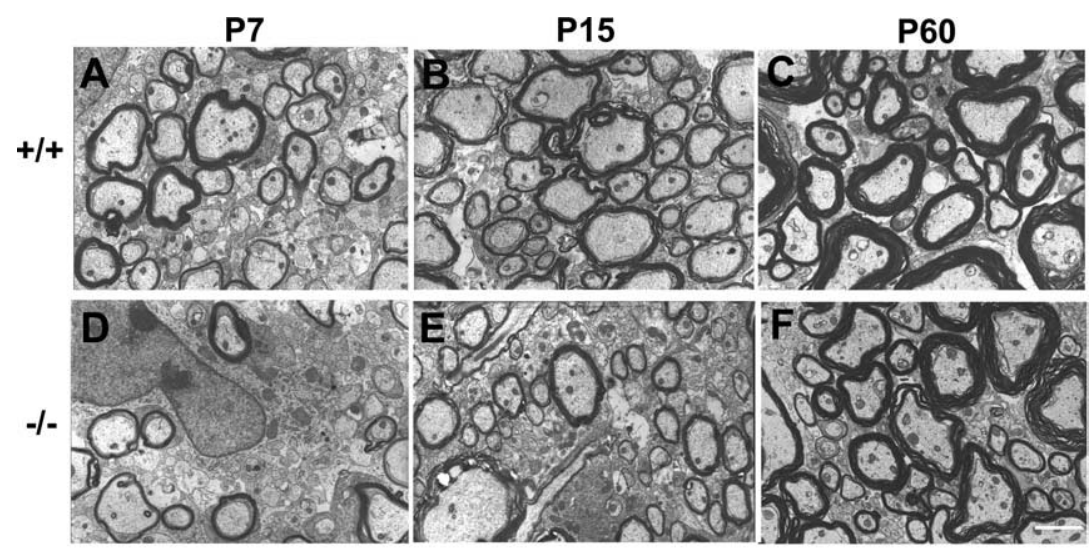

G Myelination in Necl1 KO spinal cords

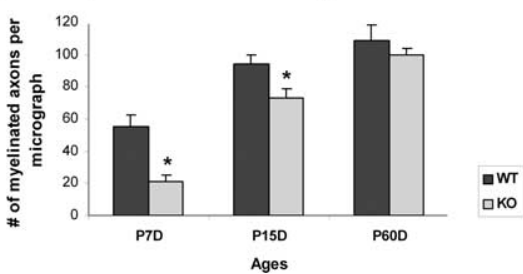

H

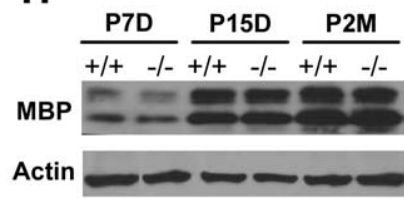

Figure 4. Delayed axonal myelination in the Necl-1 mutant spinal cords. $\boldsymbol{A}-\boldsymbol{F}$, Lateral white matter tissues from P7, P15 and P60 wild-type $(\boldsymbol{A}-\boldsymbol{C})$ and Necl-1 mutant $(\boldsymbol{D}-\boldsymbol{F})$ spinal cords were processed for EM analyses. $\boldsymbol{G}$, Number of myelinated axons per micrograph $\left(500 \mu \mathrm{m}^{2}\right)$ in the wild-type and mutant tissues at various postnatal stages $(n=3)$. Statistically significant differences $(p<0.05)$ between the wild-type and mutant animals were indicated by asterisks. Scale bar, $2 \mu \mathrm{m}$. $\boldsymbol{H}$, Western immunoblotting of P7-P60 spinal cords with anti-MBP and anti- $\beta$-actin antibodies.

displayed strong heterophilic interaction in vitro (Fogel et al., 2007; Maurel et al., 2007; Spiegel et al., 2007); and more importantly, Necl-4 expression is marked upregulated in oligodendrocytes during myelination stages (supplemental Fig S4, available at www.jneurosci.org as supplemental material). 
Intriguingly, it was recently reported that the heterophilic interaction between $\mathrm{Necl}-1$ and $\mathrm{Necl}-4$ was essential for the myelination of PNS neurons by Schwann cells in DRG neuron and Schwann cell coculture (Maurel et al., 2007; Spiegel et al., 2007). Blockade of Necl-1 and Necl-4 heterophilic binding by NECL1-Fc or NECL-4-Fc fusion protein dramatically inhibited DRG neuron myelination by Schwann cells in culture (Spiegel et al., 2007). Surprisingly, genetic disruption of Necl-1 gene did not have a significant effect on sciatic nerve myelination by Schwann cells. From P7 to P60, the percentage of myelinated axons and the thickness of myelin sheaths in null mutants were similar to those in the wild-type animals (supplemental Fig S3, available at www.jneurosci.org as supplemental material).

The discrepancies between our in vivo study and the previously reported in vitro studies (Maurel et al., 2007; Spiegel et al., 2007) could be explained by the potential functional redundancy from the related $\mathrm{Necl}$ adhesion molecules that are located on axonal membranes. For instance, Necl-3 is expressed in both the PNS and CNS neurons (Pellissier et al., 2007; Spiegel et al., 2007), and can bind to Necl-4 molecule and to oligodendrocyte or Schwann cells directly in culture (Pellissier et al., 2007; Spiegel et al., 2007). In Necl-1 mutants, $\mathrm{Necl}-4$ expression in the white matter oligodendroglial cells did not appear to be affected (supplemental Fig S4, available at www.jneurosci.org as supplemental material). It is plausible that $\mathrm{Necl}-3$ and $\mathrm{Necl}-4$ may represent another pair of interacting adhesion proteins that participate in axonal-glial adhesion and myelin ensheathment in the developing nervous system. The relatively weak interaction between axonal Necl-3 and glial Necl-4 may be sufficient to substitute the $\mathrm{Necl}-1$ and $\mathrm{Necl}-4$ binding in the initiation of myelination process in vivo in the presence of other cell adhesion interactions (Colognato et al., 2004; Maurel et al., 2007; Spiegel et al., 2007). However, these other interactions could be lost in dissociated cultures, and the weak interactions between Necl-3 and Necl-4 may not be sufficient to promote axonal myelinatin in vitro. In addition, NECL-2 protein was also expressed by axons in both the CNS and PNS (Fujita et al., 2005; Fogel et al., 2007), raising the possibility that the loss of $\mathrm{Necl}-1$ expression could be compensated by the presence of NECL-2 protein on the axonal membranes. Future studies with various $\mathrm{Necl}$ compound mutants will be needed to investigate their possible functional redundancies in axon-glial interaction and axonal myelination.

\section{References}

Biederer T (2006) Bioinformatic characterization of the SynCAM family of immunoglobulin-like domain-containing adhesion molecules. Genomics 87:139-150.

Biederer T, Sara Y, Mozhayeva M, Atasoy D, Liu X, Kavalali ET, Südhof TC (2002) SynCAM, a synaptic adhesion molecule that drives synapse assembly. Science 297:1525-1531.

Colello RJ, Pott U (1997) Signals that initiate myelination in the developing mammalian nervous system. Mol Neurobiol 15:83-100.

Colognato H, Ramachandrappa S, Olsen IM, ffrench-Constant C (2004) In- tegrins direct Src family kinases to regulate distinct phases of oligodendrocyte development. J Cell Biol 167:365-375.

Edgar JM, Garbern J (2004) The myelinated axon is dependent on the myelinating cell for support and maintenance: molecules involved. J Neurosci Res 76:593-598.

Fogel A, Akins MR, Krupp AJ, Stagi M, Stein V, Biederer T (2007) SynCAMs organize synapses through heterophilic adhesion. J Neurosci 27:12516-12530.

Fujita E, Urase K, Soyama A, Kouroku Y, Momoi T (2005) Distribution of RA175/TSLC1/SynCAM, a member of the immunoglobulin superfamily, in the developing nervous system. Brain Res Dev Brain Res 154-2:199-209.

Fukuhara H, Kuramochi M, Nobukuni T, Fukami T, Saino M, Maruyama T, Nomura S, Sekiya T, Murakami Y (2001) Isolation of the TSLL1 and TSLL2 genes, members of the tumor suppressor TSLC1 gene family encoding transmembrane proteins. Oncogene 20:5401-5407.

Kakunaga S, Ikeda W, Itoh S, Deguchi-Tawarada M, Ohtsuka T, Mizoguchi A, Takai Y (2005) Nectin-like molecule-1/TSLL1/SynCAM3: a neural tissue-specific immunoglobulin-like cell-cell adhesion molecule localizing at non-junctional contact sites of presynaptic nerve terminals, axons and glia cell processes. J Cell Sci 118:1267-1277.

Maurel P, Einheber S, Galinska J, Thaker P, Lam I, Rubin MB, Scherer SS, Murakami Y, Gutmann DH, Salzer JL (2007) Nectin-like proteins mediate axon Schwann cell interactions along the internode and are essential for myelination. J Cell Biol 178:861-874.

Miyoshi J, Takai Y (2007) Nectin and nectin-like molecules: biology and pathology. Am J Nephrol 27:590-604.

Mullen RJ, Buck CR, Smith AM (1992) “NeuN, a neuronal specific nuclear protein in vertebrates". Development 116:201-211.

Pellissier F, Gerber A, Bauer C, Ballivet M, Ossipow V (2007) The adhesion molecule Necl-3/SynCAM-2 localizes to myelinated axons binds to oligodendrocytes and promotes cell adhesion. BMC Neurosci 29-8:90.

Perlin JR, Talbot WS (2007) Putting the glue in glia: Necls mediate Schwann cell axon adhesion. J Cell Biol 178:721-723.

Sara Y, Biederer T, Atasoy D, Chubykin A, Mozhayeva MG, Südhof TC, Kavalali ET (2005) Selective capability of SynCAM and neuroligin for functional synapse assembly. J Neurosci 25:260-270.

Sarnat HB, Nochlin D, Born DE (1998) Neuronal nuclear antigen (NeuN): a marker of neuronal maturation in early human fetal nervous system. Brain Dev 20:88-94.

Schaeren-Wiemers N, Gerfin-Moser A (1993) A single protocol to detect transcripts of various types and expression levels in neural tissue and cultured cells: in situ hybridization using digoxigenin-labeled cRNA probes. Histochemistry 100:431-440.

Spiegel I, Adamsky K, Eshed Y, Milo R, Sabanay H, Sarig-Nadir O, Horresh I, Scherer SS, Rasband MN, Peles E (2007) A central role for Necl4 (SynCAM4) in Schwann cell-axon interaction and myelination. Nat Neurosci 10:861-869.

Takai Y, Irie K, Shimizu K, Sakisaka T, Ikeda W (2003) Nectins and nectinlike molecules: roles in cell adhesion, migration, and polarization. Cancer Sci 94:655-667.

Urase K, Soyama A, Fujita E, Momoi T (2001) Expression of RAI75 mRNA, a new member of the immunoglobulin superfamily, in developing mouse brain. Neuroreport 12:3217-3221.

Wakayama T, Ohashi K, Mizuno K, Iseki S (2001) Cloning and characterization of a novel mouse immunoglobulin superfamily gene expressed in early spermatogenic cells. Mol Reprod Dev 60:158-164.

Zhou Y, Du G, Hu X, Yu S, Liu Y, Xu Y, Huang X, Liu J, Yin B, Fan M, Peng X, Qiang B, Yuan J (2005) Nectin-like molecule 1 is a protein $4.1 \mathrm{~N}$ associated protein and recruits protein $4.1 \mathrm{~N}$ from cytoplasm to the plasma membrane. Biochim Biophys Acta 1669:142-154. 